\title{
Abandono de tratamento na psicoterapia psicanalítica: em busca de definição
}

\author{
Treatment dropout in psychoanalytical psychotherapy: searching for definition
}

Marina Bento Gastaud', Maria Lúcia Tiellet Nunes²

\section{RESUMO}

Objetivos: Este artigo tem por objetivo revisar as definições de abandono de tratamento encontradas na literatura sobre psicoterapia, problematizar a dificuldade de definir abandono nas diversas abordagens psicoterapêuticas, refletir sobre os critérios utilizados nas diferentes definições e propor uma definição de abandono para a psicoterapia psicanalítica. Método: Realizou-se revisão de artigos sobre abandono de tratamento envolvendo diferentes modalidades psicoterapêuticas, encontrados nas seguintes bases de dados: SciELO, Lilacs, Medline e PsycINFO. Resultados e discussão: Definições de abandono de tratamento são apresentadas em três quadros, divididos pelo referencial teórico do processo psicoterapêutico. Os critérios utilizados nas definições são discutidos. Conclusão: A fim de

\section{Palavras-chave}

Abandono, psicoterapia psicanalítica, definição.

\section{Keywords}

Dropout, psychoanalytical psychotherapy, definition. construir uma definição padronizada de abandono para psicoterapia psicanalítica, propõese três categorias de definição para término de psicoterapia, com base na discussão crítica da literatura revisada.

\section{ABSTRACT}

Objectives: This article aims to review the definitions of treatment dropout in psychotherapy literature, discuss the difficulty of defining dropout in various psychotherapeutic approaches, reflect on the criteria used in different definitions and propose a dropout definition for psychoanalytical psychotherapy. Method: A review of articles on treatment dropout involving different psychotherapeutic modalities was conducted in the following databases: SciELO, Lilacs, Medline and PsycINFO. Results and discuss: Definitions of treatment dropout are presented in three figures and divided by the theoretical framework of the psychotherapeutic process. Criteria used in definitions are discussed. Conclusion: In order to build a standardized definition of psychoanalytical psychotherapy dropout, three definition categories for termination of psychotherapy are proposed, based on the critical discussion of the reviewed literature.
1 Universidade Federal do Rio Grande do Sul (UFRGS), Ciências Médicas em Psiquiatria.

2 Pontifícia Universidade Católica do Rio Grande do Sul (PUC-RS); Tratamento e Prevenção pela Freie Universität Berlin.
Recebido em

29/5/2010

Aprovado em

$23 / 6 / 2010$
Endereço para correspondência: Marina Bento Gastaud

Rua Comendador Caminha, 312, sala 205, Bairro Moinhos de Vento 90430-030 - Porto Alegre, RS

Telefone: (51) 8428-8228

E-mail: marinagastaud@hotmail.com 


\section{INTRODUÇÃO}

O abandono de tratamento na psicoterapia vem ganhando amplo espaço de pesquisa e discussão no meio clínico e científico. As altas taxas de abandono estimadas pela literatura nacional e internacional justificam a crescente preocupação com esse fenômeno. Em 1993, em estudo de referência sobre o fenômeno, Wierzbicki e Pekarik ${ }^{1}$ demonstraram que $48 \%$ dos pacientes abandonam seus tratamentos. Estudos mais recentes conduzidos em centros de saúde mental em diversos países, dentre os quais Inglaterra, Estados Unidos e Espanha, estimaram taxas de abandono variando entre 24\% e $66 \%^{2}$. No Brasil, em estudo realizado em Belo Horizonte, Melo e Guimarães ${ }^{3}$ encontraram que 39,3\% dos pacientes que buscaram ajuda psiquiátrica abandonaram seus atendimentos. Outro exemplo brasileiro encontra-se no Rio Grande do Sul, onde Lhullier et al. ${ }^{4}$ demonstraram que $49,5 \%$ dos pacientes atendidos em uma clínica-escola de Pelotas abandonaram a psicoterapia. Luk et al. ${ }^{5}$, embora não estimem um valor específico, preconizam que as taxas de abandono chegam a ser ainda maiores na prática clínica de rotina do que em ensaios clínicos randomizados.

O abandono de tratamento na psicoterapia constitui-se em problema com graves consequências para o indivíduo e para a sociedade, tendo em vista que poucas pessoas conseguem de fato obter ajuda especializada para seu sofrimento e resolver seus problemas psicológicos. Os problemas de saúde mental atingem uma magnitude preocupante de pessoas: a última grande estimativa brasileira, na década de 1990, encontrou que entre 30\% e 50\% da população brasileira sofria de alguma sintomatologia psiquiátrica e de $20 \%$ a 35\% necessitariam de atendimento especializado em saúde mental ${ }^{6}$. A morbidade psiquiátrica na cidade de Porto Alegre foi estimada em $49 \%{ }^{7}$. Entre as pessoas que chegam à psicoterapia, os altos índices de abandono evidenciam que uma pequena parcela dos pacientes que procuram auxílio psicológico de fato chega a concluir os tratamentos que inicia. Há uma defasagem entre a necessidade de ajuda psicológica da população, a procura por atendimento especializado e os benefícios recebidos com os tratamentos psicoterápicos. As consequências do abandono mostram-se importantes tanto para os pacientes quanto para os terapeutas e serviços que prestam atendimento. Aproximadamente, todas as formas de psicoterapia requerem mais do que algumas sessões para obter sucesso, tornando a eficácia terapêutica dependente da perseverança do paciente ${ }^{8}$. Os pacientes que abandonam não se beneficiam totalmente do atendimento e acabam perdendo as esperanças de serem ajudados ${ }^{9}$. Há um alto investimento no tratamento e, quando este é interrompido antes que resultados sejam obtidos, o custo-benefício acaba sendo relativizado. Ocorre desgaste financeiro e afetivo do paciente e do profissional que atende, tendo em vista todo o investimento de ambas as partes para que se ini- cie um tratamento. Por fim, o abandono acarreta frustração para o terapeuta e para o serviço que presta o atendimento, os quais se sentem incapazes, incompetentes e desacreditados quando o paciente desiste da terapia ${ }^{5}$. Considerando que os abandonos com frequência são precedidos por não comparecimentos às sessões ou sucessivas desmarcações, os pacientes que abandonam ocupam horários dos profissionais e instituições que poderiam ser oferecidos a outros pacientes. Como resultado, a produtividade da clínica e dos profissionais fica mais reduzida e o custo dos tratamentos fica mais elevado?.

A importância de estudar o abandono da psicoterapia parece ser, portanto, inquestionável. De fato, ao pesquisar a literatura científica destinada a esse tema, percebe-se que há uma vasta lista de pesquisas visando entender o fenômeno do abandono (Tabela 1).

Grande parte dos estudos dedica-se a encontrar relações entre abandono de tratamento e características dos pacientes, dos terapeutas ou dos serviços. Preocupa-se, assim, em levantar e estimar prevalências de abandono, encontrar preditores de abandono e/ou descobrir variáveis correlacionadas, objetivando propor estratégias para minimizar as altas taxas de evasão de tratamento.

Entretanto, quando comparados entre si, os achados dessas pesquisas muitas vezes se contradizem. Isso acaba dificultando o estabelecimento de medidas preventivas, já que as conclusões e os resultados não são unânimes e apontam para nortes muito distintos. Para tomar alguns exemplos, a prevalência de abandono varia entre 15\% e 75\% $2,10,11$, uma amplitude demasiadamente abrangente para que se possam tirar conclusões mais definitivas. Citando apenas uma das contradições entre os achados, enquanto para alguns autores $^{12,13}$ altas taxas de abandono são diretamente relacionadas a baixas condições socioeconômicas, para outros pesquisadore ${ }^{14}$ altas taxas de abandono se relacionam com altas condições socioeconômicas. Há ainda uma terceira categoria ${ }^{2,15,16}$ que afirma não haver relação entre essas duas variáveis.

A qualidade do serviço prestado, a modalidade do tratamento e a amostra pesquisada variam muito entre os locais em que os estudos foram feitos. Diferenças culturais também podem estar envolvidas, já que a comparação está sendo feita entre estudos de diferentes regiões. Mesmo assim, o que torna os resultados muito diferentes também diz respeito ao método ou delineamento do estudo. A principal variável de interesse - abandono de tratamento - não está definida de forma semelhante entre as pesquisas, tornando difícil a sistematização dos resultados. Os critérios utilizados para definir o abandono são diversos e explicam, entre outros fatores, a contradição dos achados.

Dessa forma, o objetivo do presente artigo é problematizar a dificuldade de definir abandono de tratamento nas diversas abordagens de psicoterapia, refletir sobre os critérios 
utilizados nas diferentes definições encontradas na literatura nacional e internacional e propor uma definição de abandono para a psicoterapia psicanalítica.

\section{MÉTODO}

Foi realizada pesquisa nas principais bases de dados eletrônicas objetivando buscar artigos indexados nas bases de dados até maio de 2008 (Tabela 1), com os seguintes descritores: "abandono", "dropout" e "dropping out", correlacionados a "psicoterapia", "psychotherapy" e "psychological treatment".

Tabela 1. Número de estudos sobre abandono de tratamento encontrados nas principais bases de dados eletrônicas

\begin{tabular}{|c|c|c|c|c|c|}
\hline \multirow{2}{*}{$\begin{array}{l}\text { Bases de } \\
\text { dados }\end{array}$} & \multicolumn{4}{|c|}{ Descritores } & \multirow[t]{2}{*}{ Total } \\
\hline & $\begin{array}{c}\text { Dropout + } \\
\text { psychotherapy }\end{array}$ & $\begin{array}{l}\text { Dropping out + } \\
\text { psychotherapy }\end{array}$ & $\begin{array}{c}\text { Dropout + } \\
\text { psychological } \\
\text { treatment }\end{array}$ & $\begin{array}{l}\text { Abandono + } \\
\text { psicoterapia }\end{array}$ & \\
\hline SCiELO & 3 & 17 & 0 & 0 & 20 \\
\hline Lilacs & 3 & 0 & 0 & 39 & 42 \\
\hline Medline & 1.057 & 61 & 477 & 0 & 1.595 \\
\hline PsycINF0 & 419 & 118 & 10 & 3 & 550 \\
\hline Total & 1.482 & 196 & 487 & 42 & 2.207 \\
\hline
\end{tabular}

A partir da análise dos artigos encontrados, foram incluídos todos os artigos empíricos, teóricos e de revisão cujo foco principal do estudo fosse abandono de tratamento em psicoterapia. Foram excluídos estudos que, embora abordassem o abandono de psicoterapia, não tinham essa variável como tema central.

A análise englobou artigos que satisfizessem esse critério, independentemente da modalidade teórica adotada e independentemente da faixa etária dos sujeitos de pesquisa.

\section{RESULTADOS E DISCUSSÃO}

O termo "definition chaos" tem sido usado para caracterizar a literatura sobre abandono $0^{9,17}$, porque inconsistências são a regra, e não a exceção9 . Já em 1985, o estudo de Pekarik18 é exemplar para problematizar a situação. O autor apontou que pacientes que abandonaram o tratamento diferem dos que tiveram alta em 11 de 18 variáveis sobre o cliente e terapeuta quando o critério utilizado na pesquisa para definir abandono foi o julgamento do terapeuta, enquanto nenhuma diferença foi encontrada entre os dois grupos quando o critério utilizado foi a duração do tratamento. Assim, os achados das pesquisas são diretamente dependentes da definição utiliza- da e não há consenso entre os pesquisadores quanto à meIhor maneira de definir o abandono do tratamento.

Há uma grande dificuldade de definir "abandono" em psicoterapia. Primeiro, porque a definição depende da modalidade de tratamento, ou seja, do referencial teórico que embasa a técnica utilizada. Segundo, porque, mesmo dentro da mesma modalidade, não há concordância quanto aos critérios que devem compor essa definição.

Como regra geral, um marcador que diferencia as definições constitui-se no referencial teórico adotado no tratamento em questão. Mesmo assim, é possível perceber três linhas centrais presentes na maioria das definições. A definição é alicerçada: 1) no critério de "não comparecimento" a uma sessão agendada; 2) em um número estabelecido de sessões (em um ponto de corte, diretamente relacionado ao referencial teórico) ou 3) no julgamento do terapeuta. Wierzbicki e Pekarik' demonstraram que a maior parte dos estudos utiliza o segundo critério para definir abandono. Ademais, constataram que os índices de abandono variam dependendo do critério adotado; a média de abandono foi menor entre as pesquisas que adotaram o primeiro (baseado no "não comparecimento").

Tendo em vista que o objetivo do presente artigo não é fazer uma revisão sistemática da literatura, mas construir e propor uma definição padronizada de abandono em psicoterapia, não são apresentadas todas as definições encontradas na revisão dos artigos. Algumas foram selecionadas (as mais representativas dos pontos levantados) para exemplificar os argumentos apresentados na discussão.

Os quadros 1, 2 e 3, portanto, ilustram algumas definições utilizadas para "abandono de tratamento".

Definir abandono considerando o critério de não comparecimento, apesar de potencialmente ser de grande confiabilidade, é demasiadamente conservador', pois deixa de abarcar casos em que o paciente simplesmente não agenda uma próxima sessão. Mesmo indivíduos muito sintomáticos e cujos terapeutas julgam ser necessário continuar o tratamento são alocados no grupo de "alta" ou "continuadores" por esse critério ${ }^{18}$, apenas porque não deixaram de vir a nenhuma sessão.

Da mesma forma, definições alicerçadas na duração do tratamento possuem limitações inerentes, tendo em vista que um término inapropriado ou prematuro pode dar-se virtualmente após qualquer número de sessões' ${ }^{1}$. Além disso, a definição baseada no critério de um ponto de corte para o tempo de tratamento assume a hipótese de que há um número mínimo de sessões necessário para atingir os objetivos do tratamento. Essa hipótese pressupõe uma modalidade de tratamento mais estruturada, mais objetiva e menos flexível, como as psicoterapias que seguem manuais ou alguns tipos de terapia cognitiva, por exemplo. Esse critério de definição encontra obstáculos quando a terapia em questão segue uma abordagem humanista ou psicanalítica, entre outras. 
Quadro 1. Definições de abandono de tratamento em pesquisas sobre atendimentos psicológicos/psiquiátricos em geral

\begin{tabular}{|c|c|c|}
\hline Autor (ano) & Definição de "abandono" & Critério \\
\hline Corning et al. $(2007)^{19}$ & $\begin{array}{l}\text { Terapia terminada sem a concordância mútua entre paciente e terapeuta, sendo aquela encarada pelo terapeuta } \\
\text { como "recém-iniciada", "em curso" ou "não concluída" }\end{array}$ & Julgamento do terapeuta \\
\hline Melo e Guimarães $(2005)^{3}$ & $\begin{array}{l}\text { Pacientes que compareceram à entrevista inicial tiveram pelo menos uma indicação de nova consulta e não } \\
\text { retornaram em um período de } 4 \text { meses }\end{array}$ & $\begin{array}{l}\text { Não comparecimento } \\
\text { Ponto de corte }\end{array}$ \\
\hline Lhullier et al. $(2006)^{4}$ & $\begin{array}{l}\text { Quando o paciente, por decisão unilateral, com ou sem o conhecimento prévio do terapeuta, tendo comparecido a } \\
\text { pelo menos uma sessão de terapia, cessou de fazê-lo, definitivamente, independentemente do motivo que o levou } \\
\text { a isso }\end{array}$ & Julgamento do terapeuta \\
\hline Maramba e Hall $(2002)^{20}$ & Fracasso do cliente em retornar à segunda sessão & Não comparecimento \\
\hline Shuman e Shapiro $(2002)^{21}$ & Término não planejado, definido por uma decisão unilateral do paciente em interromper o tratamento & Julgamento do terapeuta \\
\hline Pelkonen et al. $(2000)^{13}$ & $\begin{array}{l}0 \text { tratamento não continuou após a fase de avaliação, ou o problema central não foi trabalhado e a necessidade de } \\
\text { atendimento ainda estava evidente }\end{array}$ & Julgamento do terapeuta \\
\hline Piper et al. (1999) 22 & $\begin{array}{l}\text { Situações nas quais o paciente realizou uma decisão unilateral contrária à expectativa inicial, representada pelo } \\
\text { contrato terapêutico, ou contrária às recomendações do terapeuta }\end{array}$ & Julgamento do terapeuta \\
\hline Richmond $(1992)^{8}$ & $\begin{array}{l}\text { Interrupção do tratamento por parte do paciente sem a concordância do terapeuta. Excluem-se os casos em que } 0 \\
\text { paciente interrompeu por razões logísticas (mudança, perda de emprego etc.) }\end{array}$ & Julgamento do terapeuta \\
\hline Garfield $(1989)^{23}$ & $\begin{array}{l}\text { Casos nos quais, após pelo menos uma sessão de iniciada a terapia, o paciente não retornou a nenhuma outra } \\
\text { sessão agendada }\end{array}$ & Não comparecimento \\
\hline Weisz et al. $(1987)^{16}$ & $\begin{array}{l}\text { Casos nos quais o tratamento clínico foi recomendado e oferecido, mas o paciente não compareceu a nenhuma } \\
\text { sessão após o acolhimento }\end{array}$ & Não comparecimento \\
\hline
\end{tabular}

Quadro 2. Definições de abandono de tratamento em pesquisas especificamente sobre psicoterapia psicanalítica

\begin{tabular}{ll}
\hline Autor (ano) & Definição de "abandono" \\
\hline Hauck et al. (2007) ${ }^{24}$ & Interrupção do tratamento - comunicada ou não - antes dos 3 meses (abandono precoce) \\
Philips et al. $(2007)^{25}$ & $\begin{array}{l}\text { Contato terapêutico com menos de } 3 \text { meses de duração entre a primeira e a última sessão, encerrado por iniciativa } \\
\text { do paciente, do terapeuta ou de ambos mutuamente }\end{array}$ \\
Zimmermann e Abreu (1994) ${ }^{26}$ & $\begin{array}{l}\text { O paciente ou o terapeuta simplesmente se retirou e os motivos, em geral, permaneceram ignorados, caso tenha } \\
\text { sido o paciente o abandonante. Os autores diferenciam abandono de interrupção (quando o paciente pôde elaborar } \\
\text { o fim do tratamento com o terapeuta, mesmo que este não esteja de acordo com seu término) e término prematuro } \\
\text { (quando o paciente se desligou antes da efetiva conclusão, quando esta já estava muito próxima, para evitar os } \\
\text { penosos sentimentos de separação) }\end{array}$ \\
\hline
\end{tabular}

Quadro 3. Definições de abandono de tratamento em pesquisas especificamente sobre psicoterapia cognitivo-comportamental

\begin{tabular}{|c|c|c|}
\hline Autor (ano) & Definição de "abandono" & Critério \\
\hline Arnow et al. $(2007)^{10}$ & $\begin{array}{l}\text { Pacientes designados a uma modalidade de tratamento, que completaram a avaliação básica, mas não comparece- } \\
\text { ram à totalidade das } 12 \text { sessões estabelecidas }\end{array}$ & Ponto de corte \\
\hline Bados et al. $(2007)^{2}$ & $\begin{array}{l}\text { Casos que interromperam o tratamento antes de completar } 14 \text { sessões, com exceção dos casos em que um acordo } \\
\text { foi estabelecido entre terapeuta e paciente em virtude de uma melhora significativa e confiável do paciente }\end{array}$ & $\begin{array}{l}\text { Ponto de corte } \\
\text { Julgamento do terapeuta }\end{array}$ \\
\hline Lincoln et al. $(2005)^{27}$ & $\begin{array}{l}0 \text { paciente iniciou um programa de tratamento intensivo ( } 30-80 \text { horas em um período de 1-3 semanas) e não } 0 \\
\text { completou }\end{array}$ & Ponto de corte \\
\hline Westbrook e Kirk $(2005)^{28}$ & Interrupção de tratamento sem a concordância prévia do terapeuta & Julgamento do terapeuta \\
\hline Luk et al. $(2001)^{5}$ & $\begin{array}{l}\text { Após pelo menos um encontro com o terapeuta, o paciente não compareceu às consultas agendadas e não retornou } \\
\text { para dar continuidade ao tratamento, apesar de convidado a fazê-lo }\end{array}$ & Não comparecimento \\
\hline
\end{tabular}

A duração mínima desses tipos de psicoterapia é em geral imprevisível e suas técnicas seguem um padrão que depende muito do funcionamento do paciente, tornando difícil estabelecer um ponto de corte único. Pontos de corte dependem da modalidade de tratamento: três meses, como propõem Hauck et al. ${ }^{24}$, pode ser considerado um abandono precoce para a psicoterapia psicanalítica, mas chega a ser a duração total de muitos tipos de psicoterapias cognitivas. Do mesmo modo, definir abandono por um número mínimo de sessões comparecidas não se mostra confiável, já que alguns 
pacientes apresentam melhora significativa em poucas sessões de tratamento, por vezes antes do esperado².

Assim, as definições de abandono fundamentadas no julgamento do terapeuta acabam se mostrando as mais confiáveis e generalizáveis, uma vez que respeitam a singularidade de cada caso e podem ser aplicadas em qualquer modalidade de tratamento. Além disso, selecionar um número de sessões comparecidas para definir o abandono ou decidir que um paciente que não comparece à sessão deve ser considerado abandonante não deixa de ser uma forma de julgamento do terapeuta'. Nesse sentido, definir abandono com base no julgamento do terapeuta parece ser a forma mais adequada. Certamente, pesquisas que utilizam essa definição acabam sofrendo críticas quanto à subjetividade da avaliação de cada terapeuta, os quais muitas vezes utilizam critérios diferentes para classificar um paciente como abandonante. Seria conveniente, portanto, criar uma definição padronizada para abandono que auxilie os terapeutas de diferentes escolas a classificar os términos dos tratamentos.

Visando propor, então, uma definição única e padronizada de abandono para os terapeutas e para os estudos vindouros, não parece confiável conceituar abandono utilizando como critério único o não comparecimento ou o estabelecimento de um número fixo de sessões, formas que já se mostraram ineficazes pelas razões apontadas. Kazdin ${ }^{9}$ propõe que três condições geralmente estão presentes na definição de abandono: 1) O paciente deixa o tratamento; 2) a decisão é tomada unilateralmente pelo paciente e 3) o terapeuta (ou equipe de trabalho) percebe a decisão como imprudente e prematura. A segunda condição é também enfatizada por muitos autores ${ }^{4,8,22,28}$, os quais preconizam em suas definições que o abandono se dá por decisão unilateral, ou seja, sem a concordância do terapeuta. Entretanto, há casos em que o terapeuta é comunicado pelo paciente de sua decisão de abandonar a terapia e, ainda assim, nada pode fazer para impedir o término dela - como nos casos em que o paciente perde sua fonte de renda ou muda-se de cidade, por exemplo. Tais casos não podem deixar de ser conceituados como abandonantes, já que a terapia não foi finalizada. Nesses casos, o terapeuta muitas vezes chega inclusive a concordar com o abandono do paciente e prepará-lo para tal, pois a interrupção pode estar sendo decorrente de uma mudança de vida positiva para o paciente. Trazendo algumas ilustrações, o terapeuta pode considerar uma interrupção do tratamento positiva quando o paciente muda-se de cidade em decorrência de uma promoção profissional, embora isso não signifique que a psicoterapia tenha trazido algum alívio para os sintomas e que o tratamento tenha sido finalizado. Casos em que o paciente não se adapta a uma modalidade terapêutica e é encaminhado pelo terapeuta a outro modelo de psicoterapia, por exemplo, também não devem ser considerados como alta. Em suma, o terapeuta pode concordar em terminar um tratamento porque o paciente relata obs- táculos externos para continuar ou refere insatisfação com o tipo de tratamento ${ }^{2}$. Casos assim não parecem entrar no grupo de pacientes cujo término se deu por "alta", tendo em vista que alguns pacientes abandonam o tratamento antes que ele tenha lhe trazido qualquer ajuda.

Por outro lado, prever exceções à definição de abandono, como fez Richmond ${ }^{8}$, baseado nos motivos que levaram o paciente a interromper, acaba abrindo um leque infinito de possibilidades que precisariam ser pressupostas, tornando menos consistente a definição. Além disso, cada terapeuta usaria um critério subjetivo para prever casos de exceção, levando clínicos e pesquisadores de volta ao ponto de coexistirem diferenças conceituais irreconciliáveis. Não obstante, definir abandono apoiado no motivo explicitado pelo paciente cria um impasse ainda maior, tendo em vista que Vargas e Nunes ${ }^{29}$ mostraram que a maioria dos pacientes não verbaliza nenhuma razão para o abandono do tratamento e, quando o faz, em geral expressa dificuldades financeiras. Na psicoterapia psicanalítica, principalmente, o motivo manifesto pelo paciente não deve ser priorizado, já que, independentemente do motivo alegado, o abandono, inconscientemente, pode expressar apenas uma resistência ao tratamento. A definição de abandono deve optar, assim, por trabalhar com o fenômeno em si, independentemente do relato do paciente e do motivo alegado, o qual pode conter alguma distorção ou viés de percepção. Não deve também levar em consideração a concordância ou não do terapeuta, já que isso levaria a uma definição imprecisa pelas razões anteriormente explicitadas.

Pensa-se, dessa forma, que a definição mais adequada de abandono deve ser fundamentada com base nos objetivos do tratamento, levando em consideração se esses foram ou não atingidos. A definição precisa de abandono, portanto, deve ser aquela que enquadra os casos em que o paciente ou o terapeuta termina a psicoterapia antes que os objetivos estabelecidos no contrato tenham sido atingidos, independentemente do motivo que levou à interrupção.

Pensar em uma definição enraizada nos objetivos e nas conquistas da terapia pressupõe o estabelecimento de um contrato terapêutico no início do processo. Terminada a fase de avaliação, em qualquer modalidade de tratamento, o terapeuta organiza um plano de tratamento a partir dos objetivos a serem atingidos. Esse plano é discutido com o paciente e, com base nele, se inicia uma psicoterapia. Assim, a terapia estaria encerrada no momento em que os objetivos traçados nesse contrato tiverem sido alcançados. Em uma psicoterapia de longa duração, como a psicoterapia psicanalítica, por exemplo, o contrato pode ser refeito ao longo do processo, estabelecendo constantemente novos objetivos. Em qualquer momento da psicoterapia, o paciente pode estar satisfeito em ter cumprido o primeiro plano de tratamento proposto e não se dispor a estabelecer novos objetivos. Por ter cumprido os objetivos previstos no contrato estabelecido, casos assim devem ser considerados como alta a partir 
dessa definição. Por consequência, casos em que o paciente obteve vários benefícios, mas interrompeu antes que os objetivos previstos tenham sido atingidos, devem ser considerados como abandono. Mesmo que o tratamento tenha trazido algum benefício, é considerado abandono se o paciente desiste sem atingir os benefícios estabelecidos no contrato?.

Devem-se antever também situações em que o paciente interrompe a psicoterapia quando esta ainda se encontra em período de avaliação, ou seja, antes que esteja claro para paciente e terapeuta seus objetivos no processo. Portanto, há a necessidade de criar uma terceira categoria para classificar o término do tratamento. Situações nas quais os pacientes interrompem o tratamento antes que os objetivos estejam definidos devem ser definidas como "não aderência".

Garfield $^{23}$ faz um entendimento similar ao proposto neste trabalho ao preconizar que abandonantes são os pacientes que interrompem a psicoterapia, e o período de avaliação deve ser considerado como anterior à psicoterapia. O abandono seria constituído por pacientes que, após iniciada a psicoterapia, interrompem o processo. O estabelecimento de um contrato terapêutico marcaria o ponto de corte entre não aderência e abandono.

Entretanto, apesar de essa distinção parecer clara e acessível na prática clínica, acaba sendo bastante confusa na realidade das pesquisas. Faltam dados muitas vezes que especifiquem se a psicoterapia foi ou não iniciada. Pesquisas com um olhar retroativo, como a pesquisa documental, encontram dificuldades de obter informações sobre em que etapa do processo terapêutico ocorreu a interrupção. Para a utilização da categoria "não aderência" nas pesquisas clínicas, parece necessário estipular um ponto de corte mais preciso e mais delimitado entre "não aderência" e "abandono". Johansson e Eklund ${ }^{30}$ diferenciam ambas as categorias com base no comparecimento à primeira sessão agendada. Silva et al. ${ }^{31}$ ampliam esse prazo e pressupõem que pacientes que permaneceram em atendimento por menos de um mês devem entrar na categoria de não aderência.

Nesse sentido, a fim de contemplar as diferentes técnicas terapêuticas e seus tempos de duração, preconizamos estipular pontos de corte distintos para cada modalidade no que se refere à categoria de "não aderência". Estudos específicos para cada modalidade devem ser realizados a fim de estipular quanto tempo de tratamento é necessário para diferenciar as categorias "abandono" e "não aderência" nos diversos referenciais teóricos existentes.

$O$ atendimento psicoterapêutico divide-se em duas fases: 1) avaliação e 2) tratamento/psicoterapia. A distinção entre não aderência e abandono depende, portanto, da fase em que a interrupção do atendimento ocorreu. Propõe-se, nesse trabalho, que o paciente seja considerado não aderente se o atendimento é interrompido na fase de avaliação e seja considerado abandonante se o atendimento é interrompido depois que o tratamento foi iniciado.
Ao receber um paciente que busca psicoterapia, cabe ao terapeuta fazer uma avaliação inicial e constatar a melhor forma de ajuda para esse paciente. Paciente e terapeuta, durante ou ao final dessa fase inicial, optam por iniciar ou não a psicoterapia. As entrevistas iniciais têm como objetivo verificar as indicações e contraindicações de psicoterapia psicanalítica para o caso em questão, dar início à construção de uma aliança de trabalho entre o par terapêutico, esclarecer os objetivos do tratamento e fazer um planejamento técnico que permita atingir os objetivos traçados ${ }^{32}$. Apenas após as entrevistas iniciais, o contrato é estabelecido e a psicoterapia é iniciada formalmente ${ }^{33}$. Luz ${ }^{34}$ define a fase inicial como "a etapa que se estende desde o primeiro contato do paciente com o terapeuta até o estabelecimento de uma aliança terapêutica sólida entre eles. Pode ocupar algumas semanas ou perdurar por meses" (p. 254).

Assim, apesar da necessidade de reconhecer e ponderar que o tempo necessário para a realização da avaliação pode variar dependendo do paciente e do terapeuta, torna-se necessário estipular um tempo médio de duração que sirva como ponto de corte para o início do processo. Na psicoterapia psicanalítica, a análise precedente permite propor a definição de não aderência conferida por Silva et al. ${ }^{31}$, a qual classifica como não aderentes os pacientes que ficaram por menos de um mês em atendimento.

Entende-se que tal período de tempo usa como critério a noção psicanalítica de aliança terapêutica, conforme as definições conferidas por Bordin ${ }^{35}$ e Luborsky ${ }^{36}$. Aliança terapêutica é por ambos definida como uma relação de colaboração entre paciente e terapeuta, com três constituintes: comprometimento entre ambos diante da tarefa, dos objetivos definidos e da formação de um vínculo de trabalho positivo. A partir dessa definição, foi construído um inventário para mensurar a aliança terapêutica, The Revised Helping Alliance Questionnaire (HAq-II), com um alto grau de confiabilidade e consistência ${ }^{37}$. Em pesquisas com objetivo de avaliar a aliança terapêutica usando o HAq-II, o questionário deve ser preenchido por terapeuta e paciente, uma vez que as sessões introdutórias de avaliação tenham terminado. Philips et al. ${ }^{25}$, pesquisando a aliança terapêutica na psicoterapia psicanalítica, consideraram que esse período de avaliação teria duração de duas a quatro sessões. Logo, entenderam que o prazo de duas a quatro sessões é o necessário para o estabelecimento da aliança terapêutica e para o início da psicoterapia. Na prática clínica, Berenstein ${ }^{38}$ e Etchegoyen ${ }^{39}$ defendem a realização de pelo menos duas ou três entrevistas antes de fechar o contrato com o paciente.

Em psicoterapia psicanalítica, diferentemente da análise, o tratamento obedece a uma frequência média de uma ou duas sessões semanais. Entende-se, portanto, que, na maioria dos casos, o prazo de quatro sessões equivale ao prazo de um ou meio mês de atendimento, conforme a frequência. Visando criar um critério único, um mês de atendimento é o prazo confiável para que a avaliação tenha sido concluída 
e a psicoterapia tenha sido iniciada. Propor o prazo de 15 dias não garantiria que a avaliação tenha sido concluída e o contrato tenha sido realizado.

Dessa forma, entende-se que o atendimento psicoterapêutico compreende a fase inicial de avaliação - a qual tem duração média de um mês - e a fase posterior de tratamento ou psicoterapia - iniciada no segundo mês de atendimento.

\section{CONCLUSÃO}

Assim, com base no acima exposto, propõem-se as seguintes categorias para o término do tratamento na psicoterapia psicanalítica:

1. Não aderência: o atendimento é interrompido na fase de avaliação da psicoterapia, ou seja, antes que os objetivos estabelecidos para o tratamento estejam claros para ambos os participantes ou em situações em que não há indicação de tratamento. Como regra geral, entende-se que a avaliação tenha duração de um mês.

2. Abandono: a psicoterapia é encerrada antes que os objetivos estabelecidos no contrato tenham sido atingidos, independentemente dos motivos que levaram o paciente ou o terapeuta a interrompê-la e independentemente de a decisão ter sido uni ou bilateral. $\mathrm{O}$ atendimento deve ter tido duração mínima de um mês para o paciente ser considerado abandonante.

3. Alta: a psicoterapia é encerrada quando os objetivos estabelecidos no contrato foram atingidos.

Na psicoterapia psicanalítica, com base na noção de aliança terapêutica, o limite de tempo de um mês foi estipulado para diferenciar o término por abandono do término por não aderência. Para as demais modalidades de psicoterapia, torna-se necessária a realização de mais estudos a fim de estipular um ponto de corte.

Problematizar a dificuldade de definir abandono é apenas o primeiro passo para entender a complexidade de fatores que o fenômeno impõe. Mais estudos precisam ser realizados a fim de testar a definição aqui proposta em diferentes contextos clínicos e de pesquisa, objetivando chegar a uma definição adequada e padronizada de abandono de tratamento na psicoterapia psicanalítica. A definição proposta nesse artigo constitui-se em um avanço inicial para a construção de uma definição útil tanto para a pesquisa quanto para a prática clínica.

\section{AGRADECIMENTOS}

As autoras agradecem à Coordenação de Aperfeiçoamento de Pessoal de Nível Superior (Capes) pelo apoio financeiro concedido para a realização deste estudo.

\section{CONFLITO DE INTERESSES}

Não há. Este artigo é derivado da dissertação de mestrado em Psicologia Clínica/PUCRS de autoria de Marina Bento Gastaud.

\section{REFERÊNCIAS}

1. Wierzbicki M, Pekarik G. A meta-analysis of psychotherapy dropouts. Prof Psychol Res Pr. 1993;24:190-5

2. Bados A, Balaguer G, Saldaña C. The efficacy of cognitive-behavior therapy and the problem of drop-out. J Clin Psychol. 2007;63(6):585-92.

3. Melo AP, Guimarães MD. Factors associated with psychiatric treatment dropout in a mental health reference center, Belo Horizonte. Rev Bras Psiquiatr. 2005;27(2):113-8.

4. Lhullier A, Nunes ML, Horta B. Preditores de abandono de psicoterapia em pacientes de clínica-escola. In: Silvares E, org. Atendimento psicológico em clínicas-escola. Campinas: Alínea; 2006, p. 229-56.

5. Luk E, Staiger P, Mathai J, Wong L, Birleson P, Adler R. Children with persistent conduct problems who dropout of treatment. Eur Child Adolesc Psychiatry. 2001;10:28-36.

6. Almeida Filho N, Mari JJ, Coutinho E, França JF, Fernandes JG, Andreoli B, et al. Estudo multicêntrico de morbidade psiquiátrica em áreas urbanas brasileiras. Rev ABP-APAL. 1992;14:93-104.

7. Busnello E, Pereira M, Knapp WP, Salgado CA, Taborda JG, Knijnik L, et al. Morbidade psiquiátrica na população urbana de Porto Alegre. J Bras Psiquiatr. 1993;42(Supl 1):55-60.

8. Richmond R. Discriminating variables among psychotherapy dropouts from a psychological training clinic. Prof Psychol Res Pr. 1992;23(2):123-30.

9. Kazdin A. Dropping out of child psychotherapy: issues for research and implications for practice. Clin Child Psychol Psychiatry. 1996;:1133-56.

10. Arnow B, Blasey C, Manber R, Constantino M, Markowitz J, Klein D, et al. Dropouts versus completers among chronically depressed outpatients. J Affect Dis. 2007;97:197-202.

11. Lester S, Harris S. Factors associated with first session nonattendance at a university-based family therapy clinic. Am J Fam Ther. 2007;35:363-76.

12. Kazdin A, Mazurick J, Bass D. Risk for attrition in treatment of antisocial children and families. J Clin Child Psychol. 1993;22:2-16

13. Pelkonen M, Marittunen M, Laippala P, Lönnqvist J. Factors associated with early dropout from adolescent psychiatric outpatient treatment. J Am Acad Child Adolesc Psychiatry. 2000;39(3):329-36

14. Singh $H$, Janes C, Schechtman J. Problem of children's treatment attrition and parent's perception of the diagnostic evaluation. J Psychiatr Treat Eval. 1982;4:257-63.

15. Target M, Fonagy P. Efficacy of psychoanalysis for children with emotional disorders. J Am Acad Child Adolesc Psychiatry. 1994;33:361-71.

16. Weisz J, Weiss B, Langmeyer D. Giving up on child psychotherapy: who drops out? J Consult Clin Psychol. 1987;55(6):916-8.

17. Armbruster P, Schwab-Stone M. Child guidance clinic drop outs: sociodemographic factors. Hosp Community Psychiatry. 1994;45:804-8.

18. Pekarik $G$. The effects of employing different termination classification criteria in dropout research. Psychotherapy. 1985;22(1):86-91.

19. Corning A, Malofeeva E, Bucchianeri M. Predicting termination type from client-therapist agreement on the severity of the presenting problem. Psychotherapy: Theory, Research, Practice Training. 2007;44(2):193-204.

20. Maramba G, Hall G. Meta-analyses of ethnic match as a predictor of dropout, utilization, and level of functioning. Cultur Divers Ethnic Minor Psychol. 2002;8(3):290-7.

21. Shuman A, Shapiro J. The effects of preparing parents for child psychotherapy on accuracy of expectations and treatment attendance. Community Ment Health J. 2002;38(1):3-16

22. Piper W, Joyce A, Rosie J, Ogrodniczuk J, McCallum M, O'Kelly J, et al. Prediction of dropping out in time-limited, interpretive individual psychotherapy. Psychotherapy. 1999:36(2):114-22 
23. Garfield S. Giving up on child psychotherapy: Who drops out? Comment on Weisz, Weiss and Langmeyer. J Consult Clin Psychol. 1989;57(1):168-9.

24. Hauck S, Kruel L, Sordi A, Sbardellotto G, Cervieri A, Moschetti L, et al. Fatores associados a abandono precoce do tratamento em psicoterapia de orientação analítica. Rev Psiquiatr RS. 2007;29(3):265-73.

25. Philips B, Wennberg, P, Wezbart A. Ideas of cure as a predictor of premature termination, early alliance and outcome in psychoanalytic psychotherapy. Psychol Psychother. 2007;80(2):229-45.

26. Zimmermann D, Abreu JR. Interrupção de psicoterapia psicodinâmica: contribuição ao seu estudo. Arq Bras Psicot Psicanal. 1994;1(1):66-74.

27. Lincoln TM, Rief W, Hahlweg K, Frank M, Witzleben I, Schroeder B, et al. Who comes? Who stays? Who profits? Predicting refusal, dropout, success and relapse in a short intervention for social phobia. Psychother Res. 2005;15(3):210-25.

28. Westbrook D, Kirk J. The clinical effectiveness of cognitive-behavior psychotherapy: outcome of a large sample of adults treated in routine practice. Behav Res Ther. 2005:43:1243-61.

29. Vargas F, Nunes ML. Razões expressas para 0 abandono de tratamento psicoterápico. Aletheia. 2003;17(18):155-8.

30. Johansson H, Eklund M. Helping alliance and early dropout from psychiatric out-patient care. Soc Psychiatry Psychiatr Epidemiol. 2006;41(2):140-7.
31. Silva V, Aguiar A, Felix F, Rebello G, Andrade R, Mattos H. Brazilian study on substance misuse in adolescents: associated factors and adherence to treatment. Rev Bras Psiquiatr. 2003;25(3):133-8.

32. Iankilevich E. Planejamento. In: Eizirik C, Aguiar R, Schestatsky S, orgs. Psicoterapia de orientação analítica. Porto Alegre: Artmed; 2005, p. 206-18.

33. Zimerman D. 0 primeiro contato. A entrevista inicial. Os critérios de analisabilidade. 0 contrato. In: Zimermam D. Manual de técnica psicanalítica - uma revisão. Porto Alegre: Artmed; 2004, p. 57-65.

34. Luz A. Fases da psicoterapia. In: Eizirik C, Aguiar R, Schestatsky S, orgs. Psicoterapia de orientação analítica. Porto Alegre: Artmed; 2005, p. 254-67.

35. Bordin E. The generalizability of the psychoanalytic concept of the working alliance. Psychotherapy. 1979;16:252-60.

36. Luborsky L. Helping alliance in psychotherapy. In: Claghorn J, ed. Successful psychotherapy. New York: Brunner/Mazel; 1976, p. 92-111.

37. Luborsky L, Barber J, Siqueland L, Johnson S, Najavits L, Frank A, et al. The Revised Helping Alliance Questionnaire (HAq-II). J Psychother Pr Res. 1996;5:260-71.

38. Berenstein I. Comentario al trabajo de David Liberman "Evaluación de las entrevistas...". Revista de Psicoanálisis. 1972;29:484-8.

39. Etchegoyen RH. A entrevista psicanalítica: desenvolvimento. In: Etchegoyen RH. Fundamentos da técnica psicanalítica. Porto Alegre: Artmed; 2004, p. 44-8. 